\title{
The Pirh2-keratin 8/18 interaction modulates the cellular distribution of mitochondria and UV-induced apoptosis
}

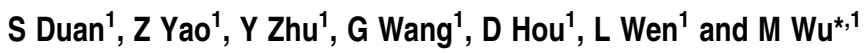

Intermediate filaments (IFs) provide crucial structural support in higher eukaryotic cells. Accumulating evidences show that IFs also participate in various cellular activities including stress responses, cell growth, cell death and cell migration through dynamic interactions with various non-structure proteins. Here we report the identification of Pirh2, a RING-H2-type ubiquitin E3 ligase, as a novel binding partner of the cytoplasmic IF proteins keratin 8/18 (K8/18). Phosphorylation of either Pirh2 or K8/18 affects their association. Although Pirh2 was not found to influence the stability of $K 8 / 18$, it displayed an unexpected role in regulating the organization of the network of $K 8 / 18$ keratin filaments. Disruption of Pirh2-K8/K18 interaction by either UV irradiation or knockdown with Pirh2 or K18 led to the aggregation of K8/18 keratin filaments. It further induced mitochondrial redistribution, and this process is likely through a microtubule-mediated pathway. The abnormal localization of mitochondria in Pirh2-knockdown cells may partially account for its increased cell sensitivity to UV-induced apoptosis, probably through enhancing the release of pro-apoptotic proteins, such as cytochrome $c$ and Smac/DIABLO to the cytosol. Overall, our data reveal the novel role of the Pirh2-K8/18 complex in governing the distribution of mitochondria.

Cell Death and Differentiation (2009) 16, 826-837; doi:10.1038/cdd.2009.12; published online 13 March 2009

The cytoskeletal network is composed of three major types of protein filaments including microtubules, microfilaments and intermediate filaments (IFs). Unlike microfilaments and microtubules, the components of which are highly conserved during evolution and are very similar to each other within the same species, the IFs consist of a large number of nuclear and cytoplasmic proteins that are expressed in a tissue- and differentiation-dependent manner. ${ }^{1-3}$ In epithelial cells, the predominant IF proteins are the keratins $(\mathrm{K})$, a multigene family of proteins that are divided into type I (K9-K20) and type II (K1-K8) subclasses. ${ }^{4}$ Members of the two subclasses form non-covalent hetero-polymers in a 1:1 ratio, and are further assembled into the keratin filaments. The expression of keratins is tissue specific, and is tightly regulated during differentiation. $\mathrm{K} 8$ and $\mathrm{K} 18$ are the major components of IFs in simple-type epithelial cells, including the liver, lung, kidney, pancreas, mammary gland, and tumors that arise from these organs. Besides their structural support for the cells, accumulating evidences have shown that K8/18 filament plays important role in modulating cellular response to apoptotic stimuli. $\mathrm{K} 8 / 18$ was reported to be responsible for cell resistance to TNF (tumor necrosis factor)-induced cytotoxicity through binding to the death receptor TNFR2 (TNF receptor type 2) and/or the adaptor molecule TRADD (TNF receptor type 1 -associated death domain protein). ${ }^{5,6}$
$\mathrm{K} 8 / \mathrm{K} 18$ was also found to confer resistance to Fas-mediated apoptosis by modulating Fas targeting to the cell surface. ${ }^{7}$ Another interesting finding reported by $M$. E. Peter's group showed that $\mathrm{K} 8 / 18$ colocalized with DEDD and active caspase-3 during apoptosis, ${ }^{8}$ implying a possible function of $\mathrm{K} 8 / 18$ in contributing to the spatial and temporal activation of caspases.

Post-translational modifications, especially phosphorylation of $\mathrm{K} 8 / 18$, have been identified to be important for the regulation of $\mathrm{K} 8 / 18$ filament organization, turnover and interaction with other proteins. ${ }^{9-11} \mathrm{~K} 8 / 18$ phosphorylation has been shown to occur during cell cycle progression or upon exposure to various growth factors or environmental stresses by stress-activated kinases, such as JNK (c-Jun N-terminal kinase) and p38 kinase. ${ }^{12,13}$

Another important role of IFs, as has been reported in recent years, is the organelle-related and protein targeting function. ${ }^{2,14}$ Studies in vimentin-knockdown mice highlighted an important link between IF proteins and the mitochondrial locations and functions. ${ }^{15,16}$ Mitochondria not only act as the cellular energy factories, but also orchestrate apoptosis by releasing pro-apoptotic factors including cytochrome $c$, Smac, Omi and apoptosis-inducing factor into cytosol and/or nucleus. ${ }^{17}$ Several studies have revealed a striking change in morphology and subcellular localization of mitochondria

${ }^{1}$ Hefei National Laboratory for Physical Sciences at Microscale and School of Life Sciences, University of Science and Technology of China, Hefei, Anhui, People's Republic of China

${ }^{*}$ Corresponding author: M Wu, School of Life Sciences, University of Science and Technology of China, huangshan Road, Hefei, Anhui 230027, People's Republic of China. Tel: + 86551 3607324; Fax: + 86551 3606264;

E-mail: wumian@ustc.edu.cn

Keywords: Pirh2; keratin 8/18; mitochondria; apoptosis

Abbreviations: IFs, intermediate filaments; TRADD, TNF receptor type 1-associated death domain protein; JNK, c-Jun N-terminal kinase; TNF, tumor necrosis factor Received 27.6.08; revised 16.12.08; accepted 31.12.08; Edited by L Scorrano; published online 13.3 .09 
during apoptosis in many cell types. ${ }^{18,19}$ Upon stimulation, mitochondria collapses from a filamentous network into punctuate fragments. The condensation of mitochondria appears to be an upstream event that contributes to the release of apoptotic factors including cytochrome $c .^{20}$

Here, we report for the first time that Pirh2, which was identified earlier as an E3 ligase that targets p53 degradation, ${ }^{21}$ is a novel K8/18-interacting partner. We have shown earlier that phosphorylation and the resultant subcellular localization of Pirh2 are important for exerting its E3 ligase activity toward $p 53 .^{22}$ However, the cytosolic function of Pirh2 and its binding partner in the cytosol has not yet been characterized. We show here that in unstressed cells, Pirh2 interacts and colocalizes with $\mathrm{K} 8 / 18$ filaments in the cytoplasm. The K8/18-Pirh2 interaction is modulated by their phosphorylation status. Binding of Pirh2 to K8/18 regulates $\mathrm{K} 8 / 18$ filament organization, which further influences the cellular distribution of mitochondria. Disruption of the interaction between Pirh2 and K8/K18, by either knockdown of Pirh2 or UV radiation, leads into an altered cellular distribution of mitochondria and enhanced UV-induced apoptosis. In addition, we also found that Pirh2-K8/18 interaction-modulated mitochondria distribution is likely through a microtubulemediated pathway. Overall, our data provided new insights into the regulation of mitochondria-dependent apoptosis through a novel pathway involving Pirh2 and K8/18.

\section{Results}

Identification of $\mathrm{K} 8 / 18$ as novel Pirh2-interacting proteins. To identify novel Pirh2-binding proteins, H1299 cell lysates were incubated with immobilized GST-Pirh2 fusion protein or GST alone, and Pirh2-interacting proteins were isolated by GST pull-down assay. As shown in Figure 1a, two major proteins of about 45 and $52 \mathrm{kDa}$ were specifically co-purified with GST-Pirh2. Mass spectrometric analysis revealed 11 and 9 peptides, respectively, from these two bands, which matched the sequences from various parts of keratins 8 and 18. To confirm this interaction, whole-cell lysates from $\mathrm{H} 1299$ cells were incubated with either GST-Pirh2 or GST alone, and samples were further analyzed by immunoblotting with anti-K8/18 antibody. As shown in Figure 1b, endogenous K8/18 was pulled down with glutathione beads only in the presence of GST-Pirh2 (lane 3, upper panel).

It has been reported that Pirh2 exhibits a dynamic distribution in both the nucleus and cytoplasm, we therefore employed immunofluorescence analysis to examine whether the cytoplasmic K8/18 filaments could influence the localization of Pirh2. In both A549 (containing wild-type p53) and H1299 (p53 null) cells, a great part of endogenous Pirh2 was found to colocalize with K8/18 filaments (Figure 1c).

To have a better understanding of which keratin, K8 or K18, binds preferentially to Pirh2, a GST pull-down assay was performed using purified GST-Pirh2 and lysates from NIH3T3 cells (which do not express endogenous K8/18) transiently expressing FLAG-tagged $\mathrm{K} 8$ and/or $\mathrm{K} 18$. As shown in Figure 1d, GST-Pirh2 was able to co-precipitate with K18 (lane 7) or K18 plus K8 (lane 8), but not with K8 alone (lane 6), suggesting that Pirh2 associated with the K8/18 polymer through direct binding to $\mathrm{K} 18$. Taken together, these results show that keratins 8 and 18 are novel Pirh2-interacting proteins.

The region of Pirh2 required for binding of $K 8 / 18$ is aa120-145. To identify the region of Pirh2 responsible for interaction with $\mathrm{K} 8 / 18$, a series of Pirh2 deletion mutants (Figure 2a) were constructed and individually co-transfected into $\mathrm{H} 1299$ cells with GFP-K8/18. The effects of the deleted domain of Pirh2 on K8/18 binding were examined by Co-IP assay. The result, as shown in Figure $2 b$ and $c$, suggested that the Pirh2 fragment spanning aa120-186 was sufficient for $\mathrm{K} 8 / 18$ binding, whereas fragment aa145-186 was unable to bind $\mathrm{K} 8 / 18$, indicating that the region of Pirh2 spanning aa120-145 was important for binding to K8/18. To confirm this finding, a Pirh2 deletion mutant lacking this region (aa120-145) was constructed and its binding affinity with K8/18 was compared with full-length Pirh2 in a Co-IP assay in $\mathrm{H} 1299$ cells. As shown in Figure $2 \mathrm{~d}$, the deletion mutant was unable to bind K8/18 (lanes 7 and 8 versus lanes 5 and $6)$. It is therefore concluded that Pirh2 aa120-145 domain is both necessary and sufficient for $\mathrm{K} 8 / 18$ binding.

Pirh2 does not affect the protein stability of keratin 8/18. An earlier report has shown that $\mathrm{K} 8$ and $\mathrm{K} 18$ could be degraded through the ubiquitin-proteasome pathway. ${ }^{10}$ However, the enzyme that carries out K8/18 ubiquitination has not yet been isolated. As Pirh2 belongs to the ring finger E3 ubiquitin ligase family, we therefore tested whether Pirh2 could target $\mathrm{K} 8 / 18$. We began this test by expressing increasing amounts of Pirh2 in $\mathrm{H} 1299$ cells stably expressing $\mathrm{K} 8$ or $\mathrm{K} 18$, and then compared the protein level of $\mathrm{K} 8$ and $\mathrm{K} 18$. As shown in Figure $3 \mathrm{~A}$, levels of $\mathrm{K} 8$ and $\mathrm{K} 18$ remained unchanged in the presence of increasingly expressed Pirh2, despite that Pirh2 itself displayed considerable auto-ubiquitination activity. To further examine the effect of endogenous Pirh2 on K8/18 protein stability, we generated a cell line carrying stably integrated shRNAi plasmid specifically against Pirh2 (Figure 3B-a) and compared the $\mathrm{K} 8 / 18$ protein level between Pirh2knockdown cell line and Pirh2 wild-type cells. Results shown in Figure 3B-b revealed that knockdown of Pirh2 did not exhibit an increase in accumulation of K8/18. Therefore, these data indicate that Pirh2, an E3 ubiquitin ligase, is not likely to be involved in the regulation of the protein stability of $\mathrm{K} 8 / 18$

UV irradiation leads to the release of Pirh2 from $\mathbf{K} 8 / \mathbf{1 8}$. To search for the role of K8/18-Pirh2 interaction in response to specific pro-apoptotic signals, H1299 cells were subjected to UV irradiation $\left(2 \mathrm{~J} / \mathrm{m}^{2}\right)$, and the interaction between Pirh2 and $\mathrm{K} 8 / 18$ was then assessed by coimmunoprecipitation. As shown in Figure 4a, UV treatment led to the disassociation between K8/18 and Pirh2 (lanes 5 and 6 versus lanes 7 and 8).

We have shown earlier that phosphorylation of Pirh2 impairs its interaction with $\mathrm{p} 53,{ }^{22}$ we are then keen to know whether the phosphorylation of Pirh2 also plays a role in regulating the association between Pirh2 and K8/18. We first 

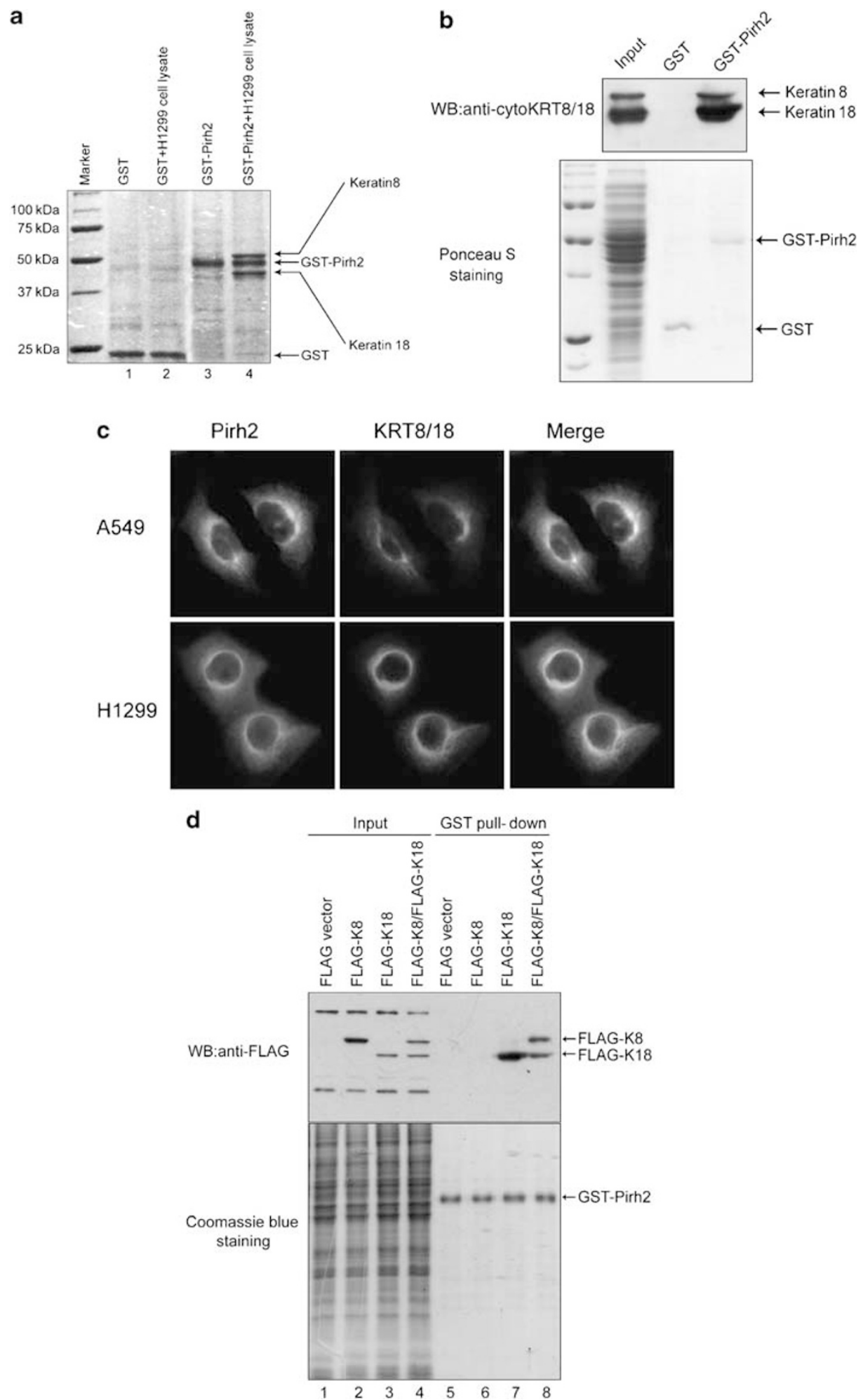

Figure 1 Identification of keratin 8/18 as novel Pirh2-interaction proteins. (a) Glutathione-agarose-bound GST protein (lanes 1 and 2) and GST-pirh2 (lanes 3 and 4 ) were incubated with and without $\mathrm{H} 1299$ cell lysate. Bead-bound proteins were washed, boiled and separated by SDS-PAGE gel. Proteins were visualized with Coomassie blue staining. The arrowheads indicate positions of GST, GST-Pirh2 and two bands that specifically co-purified with GST-Pirh2. These two bands were then analyzed by mass spectrometry and identified to be keratin 8 and keratin 18. (b) Co-immunoprecipitation of endogenous keratin 8/18 from H1299 cells with GST-tagged Pirh2. Whole-cell extract or immunoprecipitates with GST or GST-Pirh2 were analyzed by immunoblotting using anti-K8/18 antibody (upper panel). E. coli-expressed GST and GST-Pirh2 proteins were visualized by Ponceau S staining (lower panel). (c) A549 and $\mathrm{H} 1299$ cells were doubly stained with anti-Pirh2 and anti-K8/18 antibodies. The merged images are also shown. (d) E. coli-expressed and purified GST-Pirh2 was incubated with cell lysates from NIH3T3 cells expressing FLAG-tagged K8, K18 or the empty vector as indicated in the figure. Samples were then separated by SDS-PAGE, followed by western blot analysis. Equal protein loading was confirmed by Coomassie blue staining (lower panel) 


\section{a}

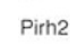

Pirh2 1.94

Pirh2 1-120

Pirh2 94-186

Pirh2 94-261

Pirh2 120-186

Pirh2 145-186

Pirh2 $\Delta 120 \cdot 145$ b

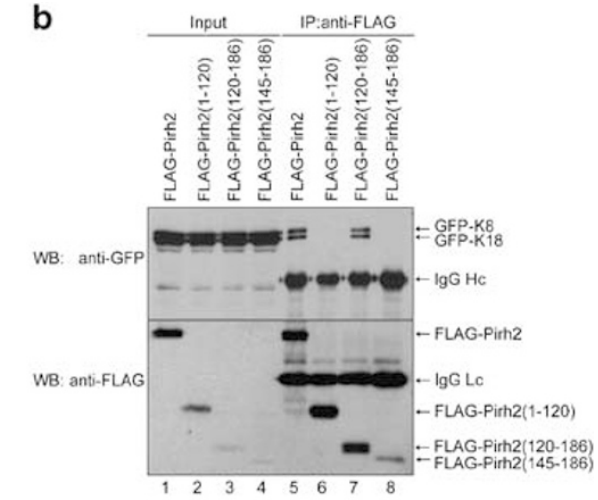

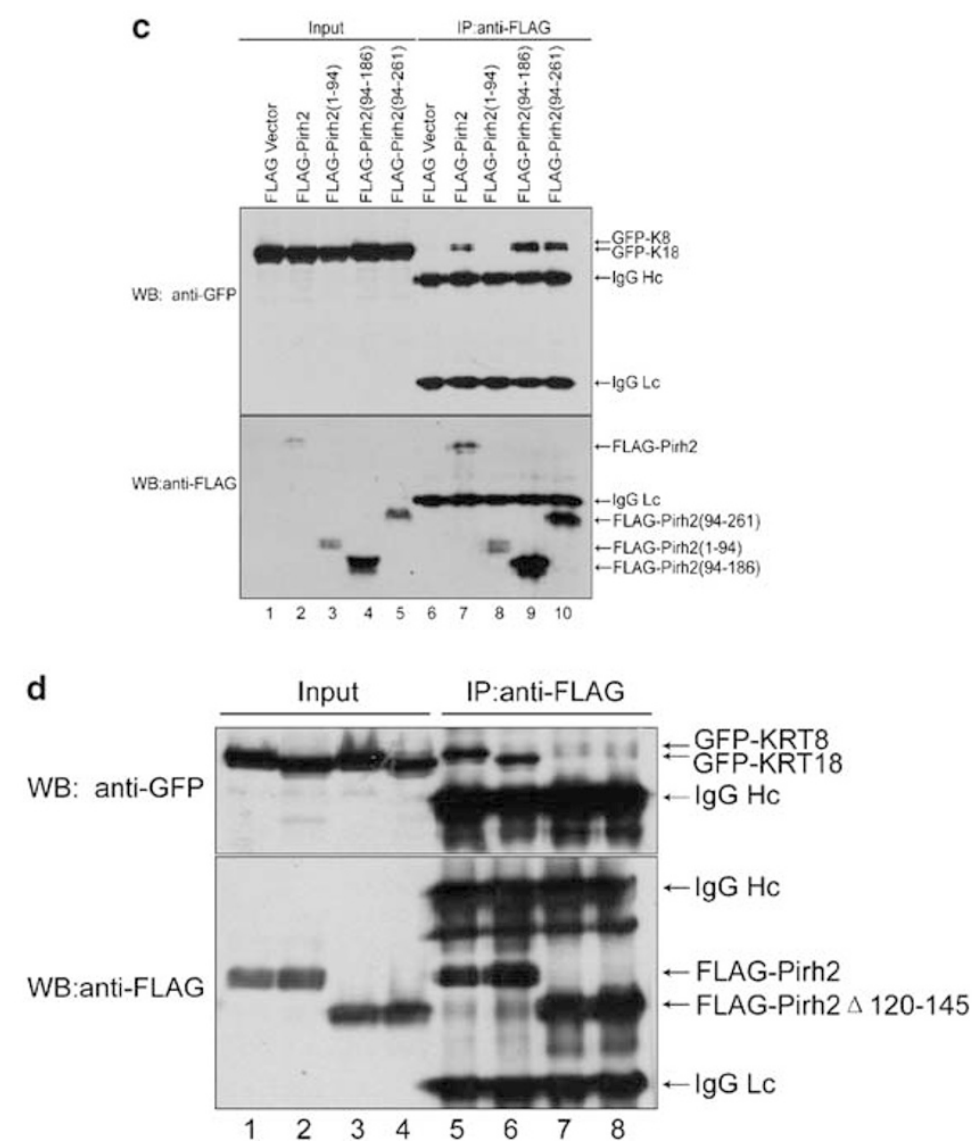

d

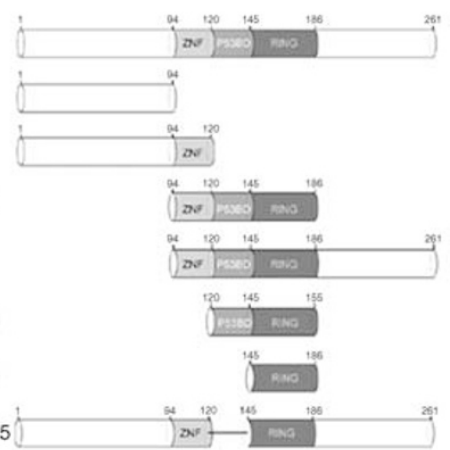

Figure 2 Mapping the domain of Pirh2 required for binding to K8/18. (a) Schematic representation of Pirh2 deletion mutants: ZNF (Zinc finger), p53-BD (p53-binding domain) and RING (RING-H2 domain). Numbers indicate the position of amino acids in Pirh2. (b and c) H1299 cells were co-transfected with a series of p3XFLAG plasmids expressing various Pirh2 deletion mutants together with pEGFP-K8 and pEGFP-K18 as indicated. After transfection, Co-IP assay was performed with anti-FLAG antibody, followed by western blot using anti-GFP and anti-FLAG antibodies. The positions of each protein are shown on the right. (d) H1299 cells were transfected with plasmids encoding FLAG-tagged Pirh2 or a Pirh2 mutant lacking aa120-145, together with plasmids expressing GFP-K8/18. Co-IP was performed as described above

compared the binding affinity between $\mathrm{K} 8 / 18$ and Pirh2 or its phosphorylated variants. Co-immunoprecipitation experiments showed that nullifying the phosphorylation of Pirh2 on both T154 and S155 significantly enhanced the interaction between K8/18 and Pirh2 (Figure 4b, lane 7 versus lane 6 ), and constitutive phosphorylation of Pirh2 greatly reduced its interaction with $\mathrm{K} 8 / \mathrm{K} 18$, as a Pirh2 phosphormimic variant Pirh2/T154ES155D failed to bind to $\mathrm{K} 8 / 18$ (lane 8 versus lane 6 ). Similar results were obtained by a reciprocal Co-IP assay in which anti-FLAG immunoprecipitates were analyzed (Figure $4 \mathrm{~b}$, lanes 9-12). Together, these data confirmed that $\mathrm{K} 8 / 18$ preferentially bound to unphosphorylated Pirh2.

The majority of Pirh2 was observed to exist as unphosphorylated proteins when cells were subjected to UV irradiation, ${ }^{22}$ which appears to be contradictory to what we just mentioned above. We then assumed that the release of Pirh2 from K8/18 upon UV irradiation might be due to 
post-translational modifications occurred on $\mathrm{K} 8 / 18$, rather than the phosphorylational status of Pirh2. K8/18 has been reported to be the target of JNK and p38 branches of the MAPK family, which are activated by UV irradiation. ${ }^{13,23,24}$ To determine whether $\mathrm{p} 38$ or JNK regulates the binding of $\mathrm{K} 8 / 18$ to Pirh2, H1299 cells were pretreated with either the p38specific inhibitor SB202190 or the JNK inhibitor SP600125 before UV irradiation exposure. As shown in Figure 4c, pretreatment of the inhibitors significantly rescued UVinduced disassociation of Pirh2 with K8/18 (lanes 5, 6 and 8, 9 versus lanes 2,3 ), suggesting that phosphorylation of $K 8 / 18$ by p38 and/or JNK is likely to be responsible for the departure of Pirh2 from K8/18.

Association between Pirh2 and K8/18 maintains K8/18 filament and mitochondrial morphology. Next, we examined whether the disassociation of Pirh2 from K8/18 could affect the organization of the K8/18 filament. As shown in Figure 5A, knockdown of Pirh2 by RNA interference in $\mathrm{H} 1299$ cells led to an overall disruption of K8/18 filament. Intriguingly, Pirh2 knockdown also exhibited a dramatic effect on the cellular distribution of mitochondria. In wild-type cells,

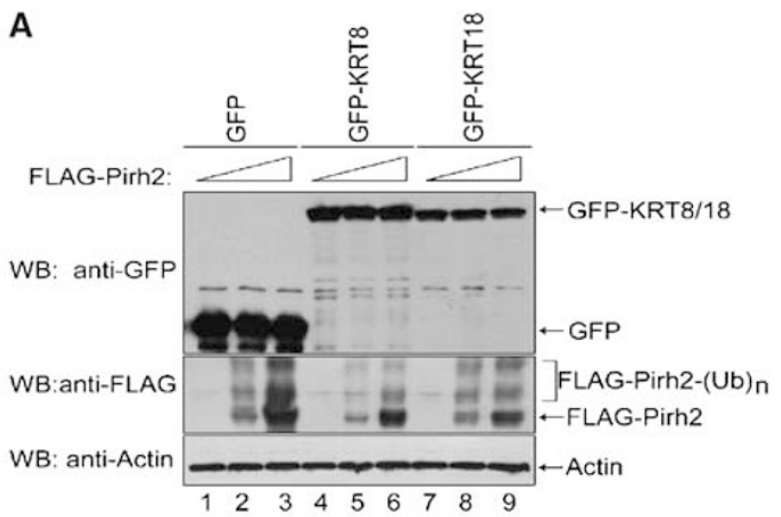

B

a

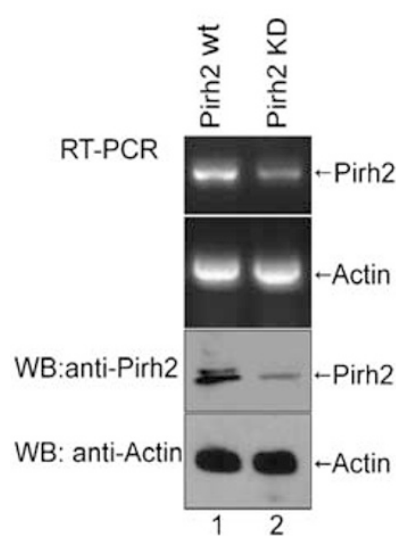

b

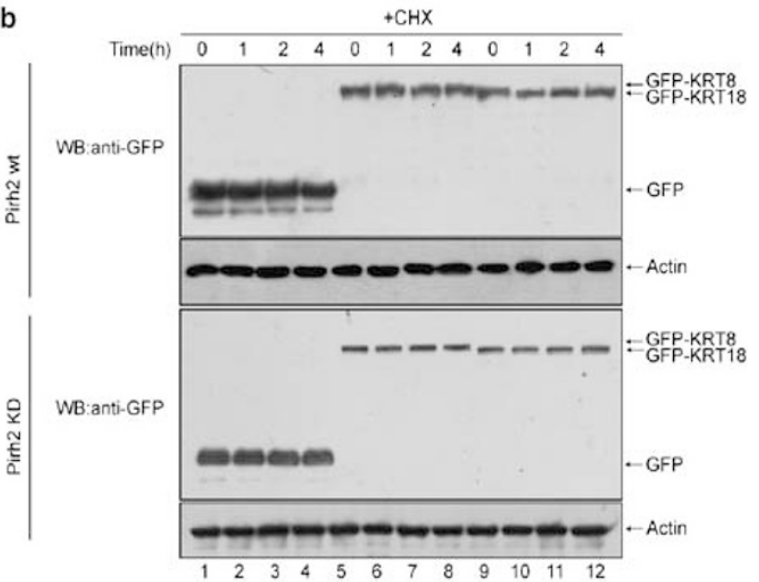

Figure 3 Pirh2 does not influence the protein stability of K8 and K18. (A) H1299 cells stably expressing GFP, GFP-K8 or GFP-K18 were transiently transfected with increasing amounts of plasmids encoding FLAG-Pirh2. Twenty-four hours after transfection, cells were collected and whole-cell extract was analyzed by immunoblotting using antibodies against GFP (upper panel) or FLAG (lower panel). $\beta$-Actin was also examined as a loading control. (B) (a) H1299 cells were infected with a retroviral-based shRNA against Pirh2 or control plasmid, and clones stably expressing the shRNA plasmids were isolated. The effect of Pirh2 knockdown was examined by reverse transcript PCR and western blotting. (b) Plasmids encoding GFP, GFP-K8 or GFP-K18 were transfected into H1299 cells stably expressing either control shRNA (upper panel) or Pirh2 shRNA (lower panel). Cells were then treated with the protein synthesis inhibitor $\mathrm{CHX}$ for the indicated times and analyzed by western blotting using anti-GFP antibody. $\beta$-Actin was also examined as a loading control

Figure 4 The interaction between Pirh2 and K8/18 is disrupted after UV irradiation (a) H1299 cells were co-transfected with plasmids expressing FLAG-Pirh2 and GFPtagged K8 or K18. The indicated transfectants were then irradiated with UV $\left(2 \mathrm{~J} / \mathrm{m}^{2}\right)$. Four hours after UV stimulation, lysates were prepared and immunoprecipitated with antiFLAG antibody. Co-precipitated GFP-K8/18 was detected with the anti-GFP antibody. (b) H1299 cells were transfected with p3XFLAG vector, or plasmids expressing wild-type Pirh2, Pirh2/S154AT155A or Pirh2/T154ES155D together with pEGFP-K8 and pEGFP-K18 as indicated in the figure. Cell lysates were immunoprecipitated with anti-GFP (lanes 4-8) or anti-FLAG (lanes 9-12) antibody and then analyzed by immunoblotting using the indicated antibodies. *Phosphorylated Pirh2. (c) H1299 cells stably expressing GFP, GFP-K8 or GFP-K18 were mock treated or pretreated with either SB202190 (an inhibitor of the p38 pathway; $10 \mu \mathrm{M}$ ) or SP600125 (an inhibitor of the JNK pathway; I $\mu$ M) for $1 \mathrm{~h}$ before irradiation by UV $\left(2 \mathrm{~J} / \mathrm{m}^{2}\right)$, and allowed to recover for $3 \mathrm{~h}$. Whole-cell lysates were then incubated with GST-Pirh2, followed by western blot analysis 
a

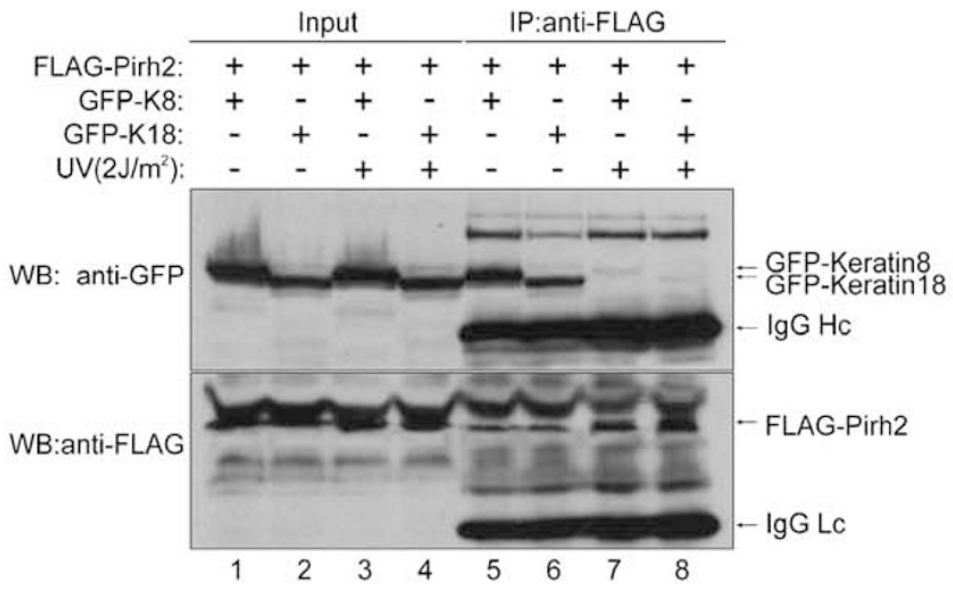

b

\begin{tabular}{|c|c|c|c|c|c|c|c|c|c|c|c|}
\hline \multicolumn{4}{|c|}{ Input } & \multicolumn{4}{|c|}{ IP:anti-GFP } & \multicolumn{4}{|c|}{ IP:anti-FLAG } \\
\hline & & $\overleftarrow{6}$ & 은 & & & 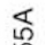 & 은 & & & 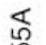 & 윰 \\
\hline & & $\frac{\infty}{\infty}$ & $=$ & & & $\frac{n}{\infty}$ & $\frac{5}{\infty}$ & & & $\frac{n}{6}$ & $\frac{10}{\infty}$ \\
\hline & & $\varangle$ & щ & & & $\ll$ & 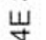 & & & $\ll$ & ঋ \\
\hline & & $\stackrel{5}{5}$ & 5 & & & $\stackrel{2}{2}$ & $\stackrel{2}{2}$ & & & เै & เै \\
\hline & & $\simeq$ & $\cong$ & 오 & $\cong$ & $\stackrel{\leftarrow}{\simeq}$ & $\stackrel{-}{\sim}$ & 옹 & $\simeq$ & $\cong$ & $\mathbb{N}$ \\
\hline$\stackrel{0}{\$}$ & 竞 & t: & 妾 & $\stackrel{0}{\$}$ & $\frac{5}{2}$ & $\frac{5}{2}$ & $\frac{5}{2}$ & ष्ष & 喜 & $\frac{5}{2}$ & $a$ \\
\hline 垈 & ర் & 安 & ర் & ত্ব & 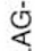 & 迆 & ญ் & ర্ষ & نே & 这 & ن் \\
\hline$\vec{u}$ & $\vec{u}$ & $\vec{u}$ & $\vec{u}$ & $\vec{\square}$ & $\overrightarrow{4}$ & $\vec{\square}$ & $\overrightarrow{4}$ & $\vec{\square}$ & $\vec{u}$ & 岌 & \\
\hline
\end{tabular}
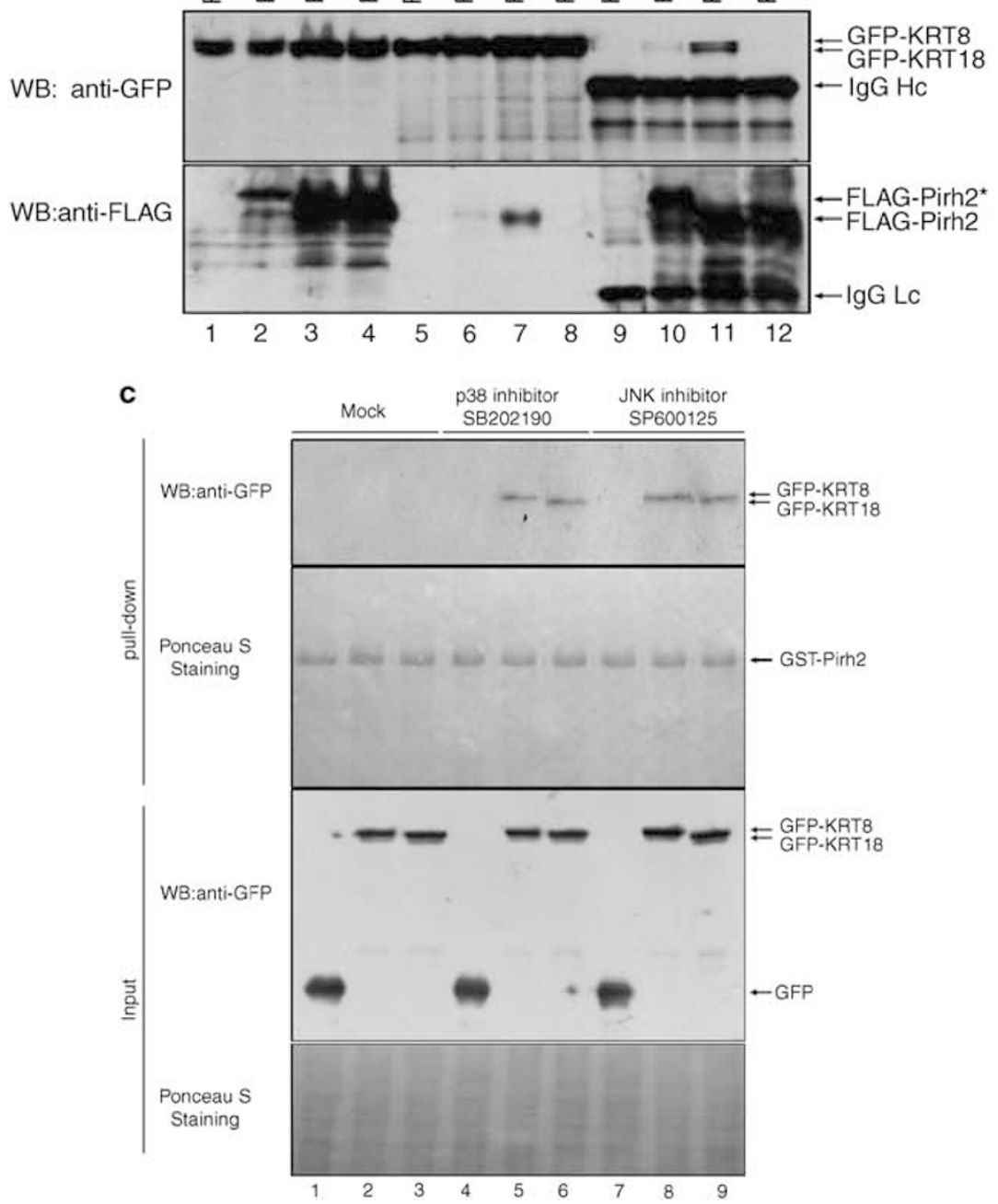
mitochondria showed a broad cytoplasmic distribution and at least partially stretched out along the K8/18 filament. In Pirh2-knockdown cells, however, the mitochondria concentrated into a juxtanuclear position that, to a great extent, colocalized with the K8/18 aggregates.

To confirm that this phenotypic effect is not due to an offtarget effect of Pirh2 RNAi, we tried to reverse the effect of Pirh2 knockdown with the ectopic expression of Pirh2 constructs. Twenty-four hours following transfection, about $70 \%$ of Pirh2-knockdown cells overexpressing the Flag-Pirh2 protein resumed normal mitochondrial morphology. In contrast, the overexpression of Flag-Pirh2 $\Delta 120-145$, which lacks the $\mathrm{K} 8 / 18$ binding site, was unable to reverse this mitochondrial aggregation (Figure 5B-a). The successful expression of FLAG-Pirh2 and Flag-Pirh2 $120-145$ in Pirh2-knockdown cells was verified by western analysis (Figure $5 B-b$ ). To further confirm that the interfering of interaction between Pirh2 and $\mathrm{K} 8 / \mathrm{K} 18$ is responsible for keratin filament disorganization and mitochondria aggregation, we knocked down the expression of K18, which was shown to directly interact with Pirh2. As shown in Figure 5C-a, similar to knockdown of Pirh2, K18 knockdown also resulted in the aggregation of both IFs and mitochondria. A double knockdown of Pirh2 and K18 displayed similar phenotype. The successful knockdown of $\mathrm{K} 18$ was verified by western analysis as shown in Figure $5 \mathrm{C}-\mathrm{b}$. These data further show that the interaction between Pirh2 and $\mathrm{K} 8 / 18$ is crucial for the organization of the $\mathrm{K} 8 / 18$ filaments and the mitochondrial network architecture.

Pirh2 and K8/18 filaments regulate mitochondrial distribution through microtubule network. To further address whether the influence of Pirh2-K8/18 on mitochondria is specific, we first examined the impact of Pirh2 knockdown on the distribution of the endoplasmic reticulum (ER) network. We observed that breaking the Pirh2 and K8/18 interaction by knocking down Pirh2 also caused redistribution of $\mathrm{ER}$, displaying similar, but not complete, overlapping images matching that of mitochondria (Figure 6a). As the major cytoskeletal elements in higher eukaryotic cells responsible for the organization of the mitochondrial and ER network were reported to be microtubules, ${ }^{25,26}$ we therefore assumed that Pirh2 may influence the distribution of these organelles through a common mechanism involving microtubules. We next explored the inter-relationships among Pirh2 and K8/18 IFs and microtubules. Under normal condition, knockdown of Pirh2 resulted in mitochondrial aggregation as shown in Figure 6b, top two panels; however, no clear differences in terms of mitochondria distributions were observed between wild-type Pirh2- and Pirh2-knockdown cells, when microtubules were either disassembled by nocodazole (bottom two panels) or stabilized by taxol (middle two panels). This observation implies that Pirh2 knockdown on mitochondria distribution may rely on microtubule architecture. In contrast, the impact of Pirh2 knockdown on $\mathrm{K} 8 / 18$ organization was unaffected by the same treatment on microtubules (Figure 6c). These data suggest that microtubules may be the downstream effector of the Pirh2-
K8/18-regulated pathway in the control of mitochondrial distribution.

Interfering of the interplay between Pirh2 and K8/18 sensitizes cells to UV-induced apoptosis. Mitochondria are of central importance in the apoptotic process. We therefore investigated whether Pirh2-K8/18-mediated alteration in the localization of mitochondria could affect cellular response to environmental stress. As shown in Figure 7A, upon UV irradiation, the $\mathrm{K} 8 / 18$ filament network collapsed and aggregated near the nucleus, exhibiting a similar pattern as was observed in Pirh2- or K18-knockdown cells. It is noteworthy that the aggregation of $\mathrm{K} 8 / 18$ filament and mitochondria preceded the nuclear condensation and fragmentation. Exposure of $\mathrm{H} 1299$ cells transfected with or without siRNA against K18 (KRT18KD2) to UV resulted in a significant mitochondrial aggregation as shown in Figure 7B. These data further confirmed that Pirh2-K8/18 disassociation was responsible for the redistribution of mitochondria in response to UV irradiation.

As we have shown above, Pirh2 disassociates from K8/18 upon UV irradiation, and pretreatment with the p38 or JNK inhibitor could prevent the loss of that interaction (Figure $4 \mathrm{a}$ and $\mathrm{c}$ ). We therefore assumed that if the loss of interaction between Pirh2 and K8/18 after UV exposure is relevant to keratin and mitochondria redistribution, pretreatment with the kinase inhibitors, which prevents Pirh2-K8/18 from disassociation, was expected to rescue, at least in part, the keratin filaments and mitochondria aggregation. This hypothesis was strongly supported by the data shown in Figure 7C. In contrast, the treatment of these inhibitors displayed no effect on Pirh2-knockdown cells, further indicating that Pirh2-K8/18 interaction is a downstream event of p38 and JNK kinase pathways.

To test whether Pirh2-K8/18-mediated mitochondrial aggregation upon UV irradiation participates in the process of apoptosis, the subcellular distributions of cytochrome $c$ and Smac were compared between wild-type $\mathrm{H} 1299$ cells and Pirh2-knockdown cells in the presence and absence of UV irradiation. As shown in Figure 7D, under normal growth conditions, cytochrome $c$ and Smac were retained in the mitochondria of both wild-type and Pirh2-knockdown cells (bottom panel, lanes 5 and 6 versus lanes 2 and 3); however, cytochrome $c$ and Smac were rapidly released from mitochondria to cytosol in Pirh2-knockdown cells in response to UV irradiation compared with that in wild-type cells (lane 12 versus lane 9). As a result, Pirh2-knockdown cells exhibited enhanced sensitivity to UV-induced apoptosis (Figure 7D). Together, these observations supported the idea that Pirh2 was required for maintaining the $\mathrm{K} 8 / 18$ filament and the proper distribution of mitochondria, which further influenced the release of mitochondrial intermembrane space proteins, including cytochrome $c$ and Smac, in response to UV irradiation.

\section{Discussion}

The present data reveal an unexpected role for the Pirh2-K8/ 18 complex in the regulation of mitochondrial distribution in 


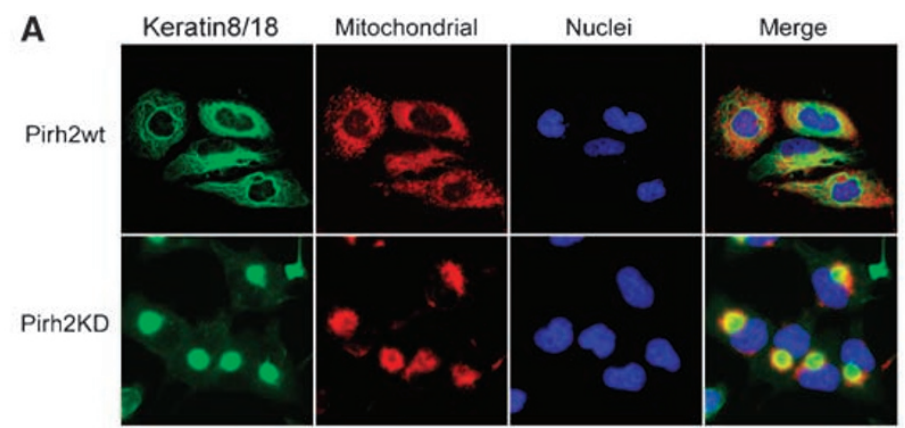

B
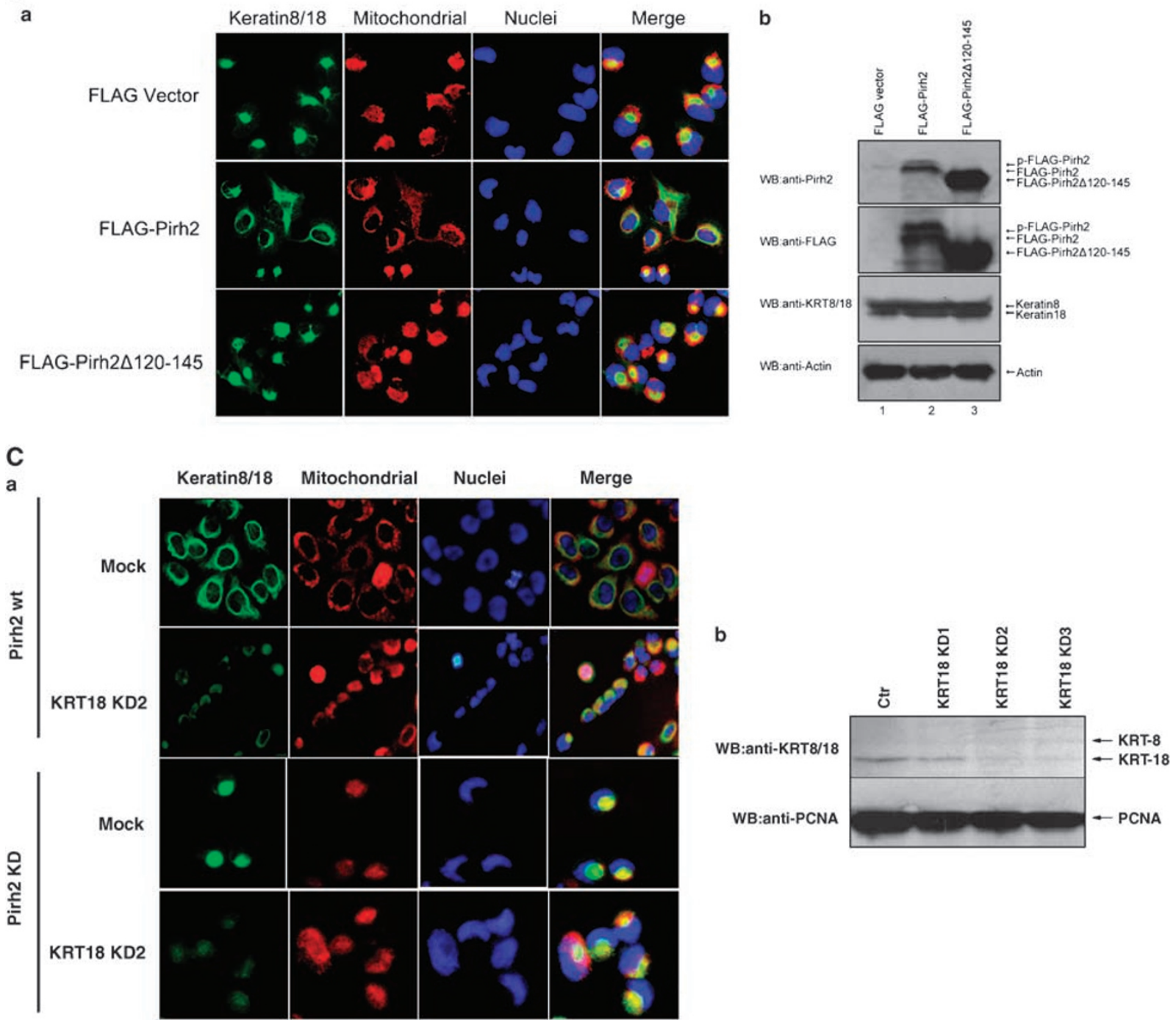

Figure 5 Pirh2 regulates the organization of the network of K8/18 keratin filaments. (A) Wild-type and Pirh2-knockdown H1299 cells were immunostained with anti-K8/18 antibody to observe the endogenous K8/18 filament. Mitochondria and nuclei were stained with Mitotracker Red and Hoechst, respectively. (B) (a) Pirh2-knockdown cells were transfected with plasmid either expressing FLAG-Pirh2, mutant FLAG-Pirh2 lacking aa120-145 or the empty vector. The endogenous K8/18 filament was then analyzed by indirect immunofluorescence. The distribution of mitochondria was visualized by staining cells with the mitochondria-specific probe MitoTracker Red. The nuclei were visualized by Hoechst staining. A merged version is also shown. (b) The expression of ectopically expressed proteins and endogenous K8/18 was analyzed by western blotting. $\beta$-Actin serves as a loading control. (C) (a) Wild-type and Pirh2-knockdown H1299 cells were mock treated or treated with RNAi against keratin 18. Forty-eight hours after transfection, mitochondria and nuclei were stained with Mitotracker Red and Hoechst, respectively. The merged image is shown on the right. (b) H1299 cells were transfected with three different siRNA against keratin 18 respectively. Forty-eight hours after transfection, the effects of three keratin 18 siRNA knockdown were examined by western blotting. PCNA was used as a loading control 


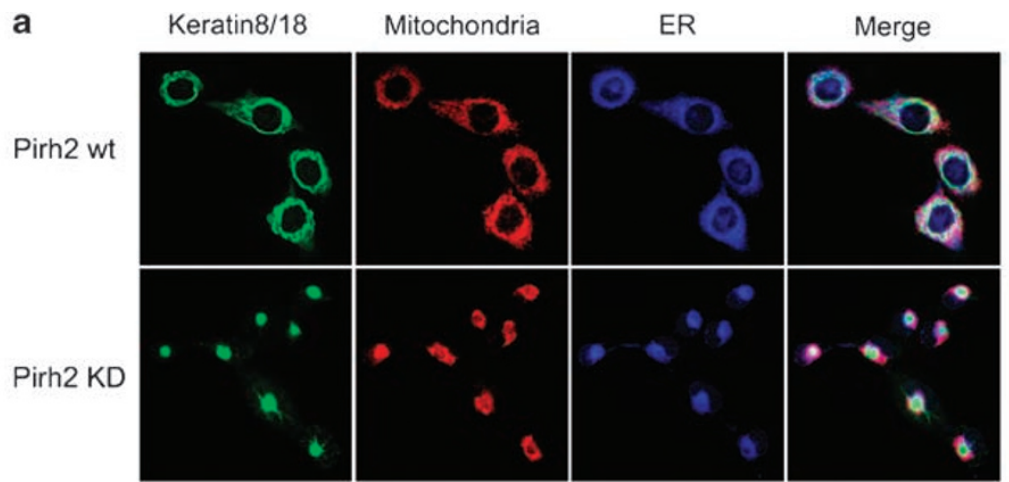

b

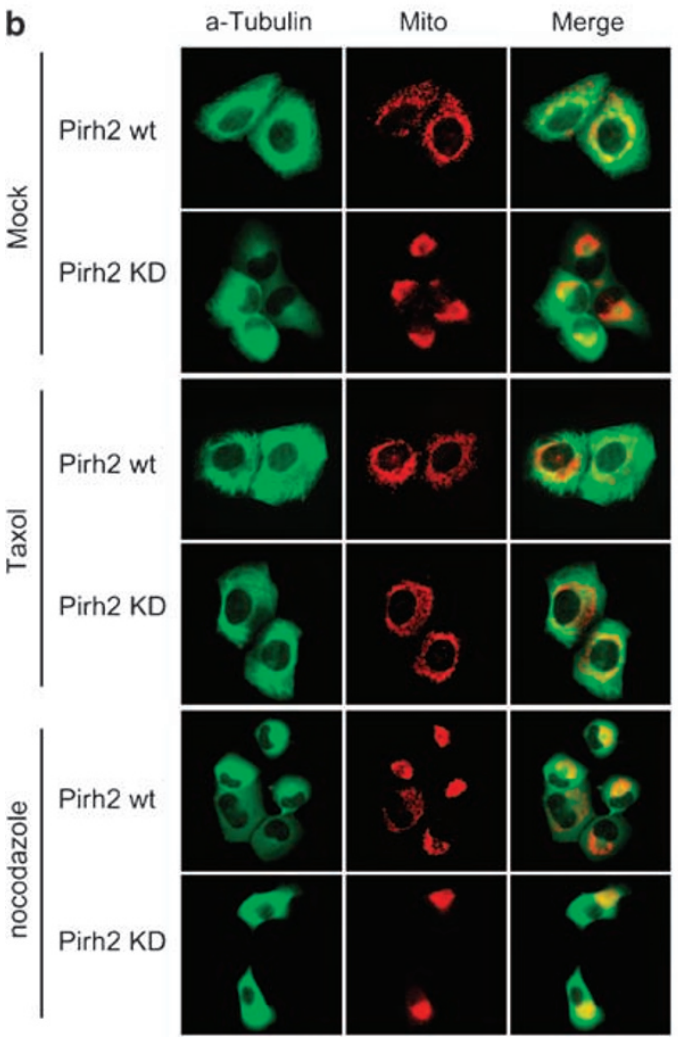

C

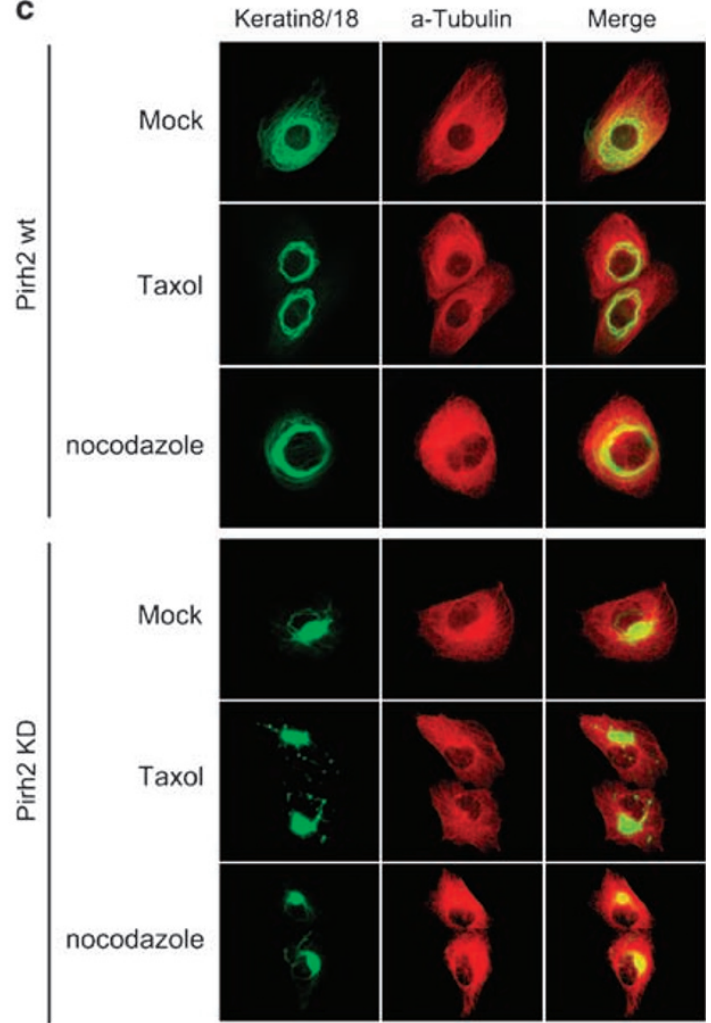

Figure 6 Re-distribution of mitochondria resulted from K8/K18 aggregation is likely through a microtubule-dependent mechanism. (a) K8/18 filament, mitochondria and ER distribution in wild-type and Pirh2-knockdown H1299 cells was analyzed by immunostaining. (b) Wild-type and Pirh2-knockdown H1299 cells expressing GFP-fused $\alpha$-tubulin were either untreated, or treated with taxol $(50 \mathrm{nM})$ or nocodazole $(250 \mathrm{ng} / \mathrm{ml})$ for $2 \mathrm{~h}$, and analyzed for GFP fluorescence using fluorescent microscopy. Mitochondria were visualized in living cells by MitoTracker staining. (c) Wild-type and Pirh2-knockdown $\mathrm{H} 1299$ cells were either untreated or pretreated with taxol or nocodazole. The endogenous K8/18 and microtubule network was visualized using anti-K8/18 antibody and anti- $\alpha$-tubulin antibody, respectively. The merged image is shown in the right panel

living cells. First, the specific interaction between Pirh2 and $\mathrm{K} 8 / 18$ was observed in unstressed cells and appears to be important for the organization of the K8/18 interfilament network. Knockdown of Pirh2 resulted in a clear aggregation for K8/18 in a manner similar to the effect of UV irradiation. As $\mathrm{K} 8 / 18$ is abundant in simple epithelial cells and associated with many proteins, it is intriguing, yet still mysterious, why the deletion of Pirh2 has such a robust effect on K8/18 filament structure. Further investigation is needed to test the underlying mechanism of how Pirh2 regulates keratin assembly and/or disassembly under different growth conditions. In addition, K8/18 also serves as a cytoplasmic anchor for Pirh2, which otherwise will translocate into the nucleus upon
UV irradiation. Interestingly, we have found that $\mathrm{K} 8 / 18$ preferentially binds to unphosphorylated Pirh2 (Figure $4 \mathrm{~b}$ ). This finding appears to be contradictory to what we have shown before. We have shown earlier that unphosphorylated Pirh2 enters the nucleus more readily than its phosphorylated counterpart. ${ }^{22}$ This dilemma can be explained by the fact that dissociating Pirh2 from K8/18 is dependent upon not only the phosphorylation of Pirh2, but also the phosphorylation of $\mathrm{K} 8 / 18$. This notion was supported by the observation that utilization of kinase inhibitors against JNK or p38 could effectively prevent the loss of interaction between Pirh2 and $\mathrm{K} 8 / 18$ upon UV irradiation (Figure 4c). Further identification of the exact phosphorylation sites on $\mathrm{K} 8$ or 18 by these different 
A

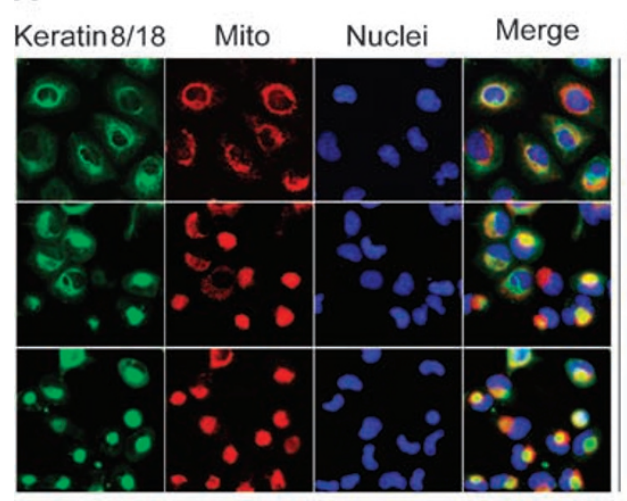

\section{B}

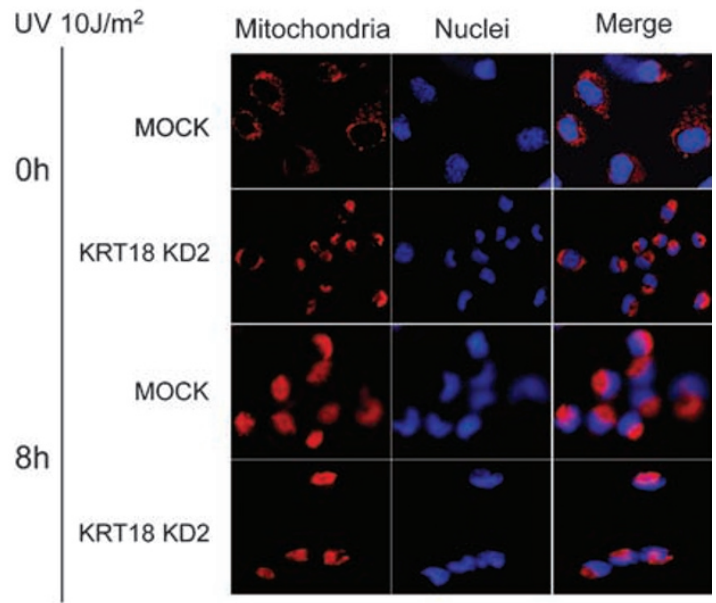

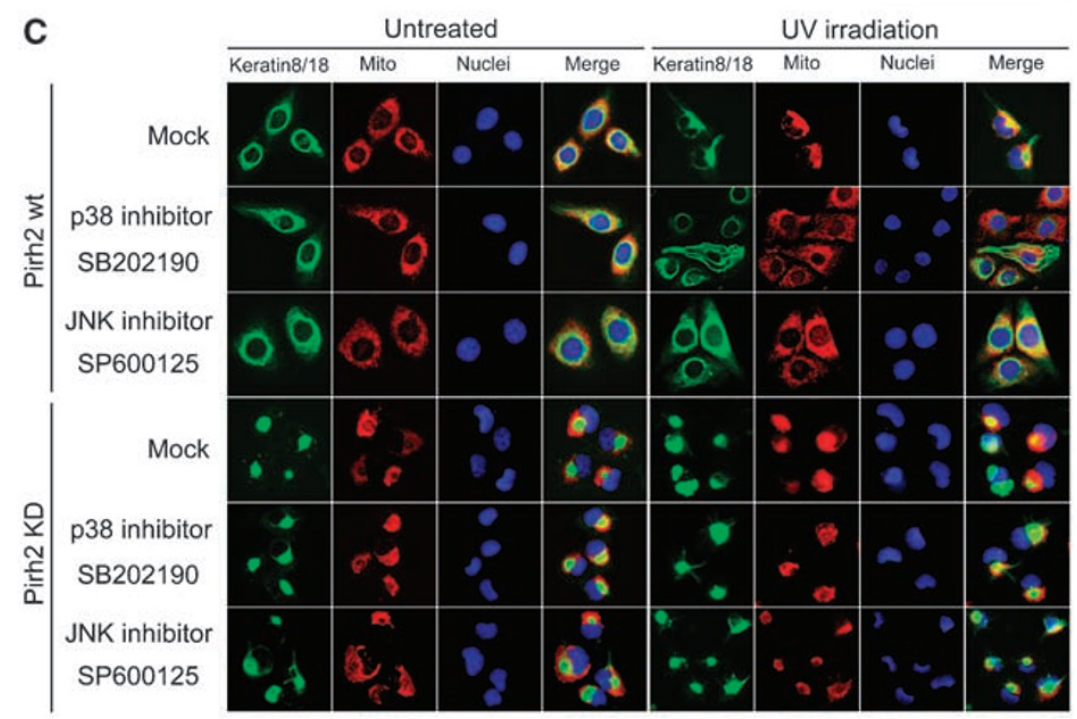
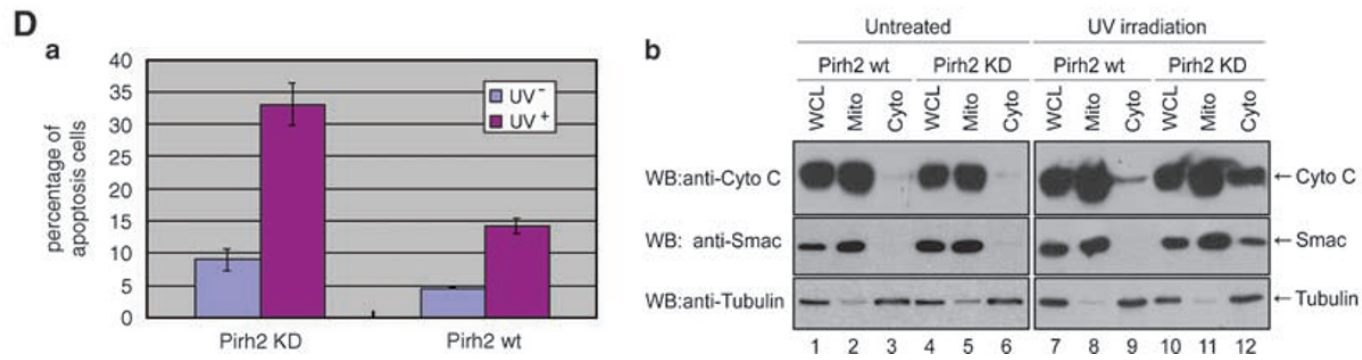

Figure 7 Disruption of Pirh2 and K8/18 leads to an enhanced UV-induced apoptotic cell death. (A) H1299 cells were subjected to UV irradiation and then allowed to recover for the indicated times. The K8/18 filament was observed by immunostaining using anti-K8/18 antibody. Mitochondria and nuclei were stained with MitoTracker and Hoechst 33342, respectively. (B) H1299 cells were transfected with or without siRNA against K18 (KRT18KD2) for $48 \mathrm{~h}$ before UV irradiation (10 J/m²), and then allowed to recover in fresh medium for $8 \mathrm{~h}$. Mitochondria and nuclei were stained with MitoTracker and Hoechst 33342, respectively. (C) Wild-type and Pirh2-knockdown H1299 cells were either mock treated, or pretreated with the p38 kinase inhibitor SB202190 $(10 \mu \mathrm{M})$ or the JNK inhibitor SP600125 $(10 \mu \mathrm{M})$. Cells were then either untreated or subjected to UV irradiation. The endogenous K8/18 filament was visualized by immunostaining using anti-K8/18 antibody. MitoTracker and Hoechst 33342 were used for mitochondrial and nuclear staining, respectively. (D) Wild-type and Pirh2-knockdown $\mathrm{H} 1299$ cells were subjected to UV irradiation $\left(10 \mathrm{~J} / \mathrm{m}^{2}\right)$, and then allowed to recover in fresh medium for $4 \mathrm{~h}$. (a) Measurement of apoptosis was based on condensed/fragmented nuclei stained with Hochest 33342 under microscopy. The percentage of apoptotic cells was determined by counting more than 300 cells in at least three separate microscopic fields. Experiment was performed in triplicate and data are represented as mean \pm standard. (b). Whole-cell extract, the mitochondrial pellet and the cytoplasmic fraction were prepared and probed with anti-cytochrome $c$ and anti-Smac antibodies. $\beta$-Tubulin was used as a cytoplasmic marker. An equal amount of protein was loaded from each fraction

kinases will contribute to a deeper understanding of the dynamic interaction between Pirh2 and $\mathrm{K} 8 / 18$ in various signaling pathways.
A significant physiological relevance of Pirh2-K8/18 interaction was reflected by the subcellular distribution of mitochondria. Disruption of the Pirh2-K8/18 complex by 
knocking down Pirh2 led to nuclear clustering of mitochondria and the formation of mitochondria aggregates, thus to enhance UV-induced apoptosis. Although the detailed mechanisms underlying these still remain to be determined, our current data revealed that the functional Pirh2-K8/18 complex may influence mitochondria distribution in a microtubule-dependent pathway. Exposure of cells to nocodazole, a chemotherapeutic drug that depolymerized microtubules, caused mitochondrial aggregation in the same pattern as that observed in Pirh2-knockdown cells (Figure 6b). Besides, the microtubule network was partially collapsed in Pirh2-knockdown cells. On the contrary, treatment with taxol, which stabilized the microtubules, prevented the clustering of mitochondria in Pirh2-knockdown cells (Figure 6b). Furthermore, no direct interaction between Pirh2 and tubulin was detected (data not shown). On the basis of these experimental observations, together with the many reported evidences of direct interconnection between the IF and microtubules, ${ }^{27}$ one can assume that Pirh2 may exert its influence on mitochondria through affecting the $\mathrm{K} 8 / 18$ filament and subsequently the microtubule network by an as-yet-unidentified mechanism.

Given the fact that $\mathrm{K} 8 / 18$ mutation or deletion is often used as a predisposing marker for cirrhosis and liver disease progression, ${ }^{28,29}$ and moreover, changes in mitochondria morphology and distribution had been reported in several liver diseases, ${ }^{30-32}$ investigations on the mechanism of how Pirh2-K8/18 interaction affects mitochondria dynamics could be an imperative research topic. A recent report demonstrated a more direct link between keratin mutation and mitochondria abnormality in liver-derived epithelial cells. ${ }^{33}$ Therefore, the functional correlations between Pirh2-K8/18 and their effect on multiple mitochondrial functions (e.g., apoptosis, energy metabolism etc) are well worthy of exploration.

\section{Materials and Methods}

Cell lines and plasmids. Human lung cancer lines H1299 and A549 were cultured in DMEM medium with $10 \%$ fetal bovine serum using standard approaches. Transient transfection was performed with Lipofectamine 2000 (Invitrogen, Eugene, OR, USA) according to the manufacturer's manual. To inhibit Pirh2 expression in human cell lines by RNA interference, double-stranded oligonucleotide targeting the sequence of Pirh2 was cloned into the pSuperRetroPuro vector (OligoEngine, Seattle, WA). The control shRNA was constructed using primers $\mathrm{P} 1$ and $\mathrm{P} 2$, and the effective Pirh2-shRNA was constructed using primers $\mathrm{P} 3$ and $\mathrm{P} 4$.

P1: 5'-GATCCCCTCCACTGTTCAGTTTCATATTCAAGAGATATGAAACTGAA CAGTGGATTTTTA-3', P2: 5'-AGCTTAAAAATCCACTGTTCAGTTTCATATCTCT TGAATATGAAACTGAACAGTGGAGGG-3'， P3: $5^{\prime}$-GATCCCCTTCAACATGCC CAACAGACTTCAAGAGAGTCTGTTGGGCATGTTGAATTTTTA-3', P4: 5'-AGCT TAAAAATTCAACATGCCCAACAGACTCTCTTGAAGTCTGTTGGGCATGTTGAA GGG-3'

To inhibit keratin 18 expression in $\mathrm{H} 1299$ cells by RNA interference, the siRNA sequences targeting K18 used were the following, of which KRT18KD2 and KRT18KD3 were effective.

KRT18KD1: UCUCAGGACCUCGCCAAGATT/UCUUGGCGAGGUCCUGAG ATT, KRT18KD2: UACAGAUGGAGCAGCUCAATT/UUGAGCUGCUCCAUCU GUATT and KRT18KD3: UGGCGAGGACUUUAAUCUUTT/AAGAUUAAAGUC CUCGCCATT.

Cells were infected with the shRNA retrovirus and selected with $2 \mu \mathrm{g} / \mathrm{ml}$ puromycin. The cell clones stably expressing Pirh2-shRNA were isolated and expanded for analysis. Wild-type Pirh2 and its mutants were cloned into mammalian and $E$. coli expression plasmids as described earlier. ${ }^{22}$ The full-length cDNA fragment coding for keratin 8 was obtained by RT-PCR using primers $5^{\prime}$-ggaattccatgtccatcagggtgacc- $3^{\prime}$ (forward) and $5^{\prime}$-gcgtcgactcacttgggcaggacgtc- $3^{\prime}$ (reverse). To amplify full-length keratin $18 \mathrm{cDNA}$, primers $5^{\prime}$-ggaattccatgagcttcac- cactcgc-3' (forward) and $5^{\prime}$-gcgtcgacttaatgcctcagaacttt-3' (reverse) were used. The resultant PCR product was subcloned into either the pEGFP-C1 (Clontech, Palo Alto, CA, USA) or the p3 $\times$ FLAG-myc-CMVTM-24 (Sigma, St. Louis, MO, USA) vector for expression in mammalian cells.

Antibodies and reagents. The following antibodies were used in this study: monoclonal anti-K8/18 antibody (Santa Cruz Biotechnology, Santa Cruz, CA, USA), mouse monoclonal anti-FLAG antibody (Sigma), rabbit polyclonal Pirh2 antibody (Bethyl, Montgomery, TX, USA), monoclonal anti-Smac antibody (Calbiochem, La Jolla, CA, USA) and monoclonal anti-cytochrome $c$ antibody (R\&D systems, Minneapolis, MN, USA)

The p38 kinase inhibitor SB205823, the JNK kinase inhibitor SP600125 and protein synthesis inhibitor $\mathrm{CHX}$ were purchased from Sigma. Mitotracker Red was obtained from Molecular Probes (Molecular Probes, Invitrogen).

Subcellular fractionation analysis. Cells were washed with phosphatebuffered saline (PBS), resuspended in homogenization buffer $(0.25 \mathrm{M}$ sucrose/ $20 \mathrm{mM}$ HEPES, pH 7.4/10 mM KCl/1 mM EGTA/1 mM EDTA/ $1 \mathrm{mM} \mathrm{MgCl} / 1 \mathrm{mM}$ DTT/the protease inhibitor cocktail) and subjected to 40 strokes in a 2-ml Dounce homogenizer on ice. Membrane rupture of cells is confirmed by microscopy observation. The homogenates were then subjected to centrifugation at $500 \times g$ for $5 \mathrm{~min}$ at $4^{\circ} \mathrm{C}$. The resulting low-speed pellet, which contained nuclei and unbroken cells, was discarded. The post-nuclear supernatant was further centrifuged at a high speed for $30 \mathrm{~min}$ at $4^{\circ} \mathrm{C}$. The resulting heavy-mitochondria pellet was resuspended in homogenization buffer containing $1 \%$ Triton X-100. The cytosolic fraction was obtained by centrifugation of the supernatant for another $30 \mathrm{~min}$ at $4^{\circ} \mathrm{C}$.

Immunofluorescence microscopy. Cells were cultured in 24-well chamber slides. After washing once with pre-warmed PBS, cells were fixed immediately in $50 \%$ methanol and $50 \%$ acetone $(\mathrm{v} / \mathrm{v})$ at $-20{ }^{\circ} \mathrm{C}$ for $10 \mathrm{~min}$. Fixed cells were washed three times with PBS before blocking with $5 \%$ bovine serum albumin in PBS for $0.5 \mathrm{~h}$, followed by incubating with the primary antibody for $1 \mathrm{~h}$ at room temperature. Immune complexes were then stained with goat anti-mouse immunoglobulin $G$ conjugated with fluorescein or rhodamine antibody (Jackson ImmunoResearch). Mitochondria were stained with MitoTracker Red. Hoechst 33342 was used for nuclear staining. Stained cells were mounted with Vectashield Mounting medium (Vector Laboratories, Burlingame, CA, USA) and analyzed under using an Olympus IX71 inverted microscope coupled to an Olympus DP70 highresolution color camera.

Acknowledgements. We thank Dr. M Bishr Omary for providing plasmids encoding the keratin $8 \mathrm{cDNA}$. This research was supported by grants from the National Natural Science Foundation of China (30530200, 30728003 and 30871290), the Ministry of Science and Technology of China (2006AA02Z101, 2006CB933300 and 2006CB910300) and the Chinese Academy of Sciences (KSCX1-YW-R-57).

1. Fuchs $E$, Cleveland DW. A structural scaffolding of intermediate filaments in health and disease. Science 1998; 279: 514-519.

2. Coulombe PA, Wong P. Cytoplasmic intermediate filaments revealed as dynamic and multipurpose scaffolds. Nat Cell Biol 2004; 6: 699-706.

3. Ku NO, Zhou X, Toivola DM, Omary MB. The cytoskeleton of digestive epithelia in health and disease. Am J Physiol 1999; 277: G1108-G1137.

4. Fuchs $E$, Weber K. Intermediate filaments: structure, dynamics, function, and disease. Annu Rev Biochem 1994; 63: 345-382.

5. Inada H, Izawa I, Nishizawa M, Fujita E, Kiyono T, Takahashi T et al. Keratin attenuates tumor necrosis factor-induced cytotoxicity through association with TRADD. J Cell Biol 2001; 155: 415-426.

6. Caulin C, Ware CF, Magin TM, Oshima RG. Keratin-dependent, epithelial resistance to tumor necrosis factor-induced apoptosis. J Cell Biol 2000; 149: 17-22.

7. Gilbert S, Loranger A, Daigle N, Marceau N. Simple epithelium keratins 8 and 18 provide resistance to Fas-mediated apoptosis. The protection occurs through a receptor-targeting modulation. J Cell Biol 2001; 154: 763-773.

8. Lee JC, Schickling O, Stegh AH, Oshima RG, Dinsdale D, Cohen GM et al. DEDD regulates degradation of intermediate filaments during apoptosis. J Cell Biol 2002; 158: 1051-1066.

9. Ku NO, Liao J, Omary MB. Phosphorylation of human keratin 18 serine 33 regulates binding to $14-3-3$ proteins. EMBO J 1998; 17: 1892-1906. 
10. Ku NO, Omary MB. Keratins turn over by ubiquitination in a phosphorylation-modulated fashion. J Cell Biol 2000; 149: 547-552.

11. Coulombe PA, Omary MB. 'Hard' and 'soft' principles defining the structure function and regulation of keratin intermediate filaments. Curr Opin Cell Biol 2002; 14: 110-122.

12. Feng L, Zhou X, Liao J, Omary MB. Pervanadate-mediated tyrosine phosphorylation of keratins 8 and 19 via a p38 mitogen-activated protein kinase-dependent pathway. J Cell Sci 1999; 112 (Part 13): 2081-2090.

13. He T, Stepulak A, Holmstrom TH, Omary MB, Eriksson JE. The intermediate filament protein keratin 8 is a novel cytoplasmic substrate for c-Jun $\mathrm{N}$-terminal kinase. J Biol Chem 2002; 277: 10767-10774.

14. Toivola DM, Tao GZ, Habtezion A, Liao J, Omary MB. Cellular integrity plus: organellerelated and protein-targeting functions of intermediate filaments. Trends Cell Biol 2005; 15 608-617.

15. Li Z, Colucci-Guyon E, Pinçon-Raymond M, Mericskay M, Pournin S, Paulin D et al. Cardiovascular lesions, skeletal myopathy in mice lacking desmin. Dev Biol 1996; 175 362-366.

16. Milner DJ, Weitzer G, Tran D, Bradley A, Capetanaki Y. Disruption of muscle architecture and myocardial degeneration in mice lacking desmin. J Cell Biol 1996; 134: 1255-1270.

17. Green DR, Kroemer G. The pathophysiology of mitochondrial cell death. Science 2004 305: 626-629.

18. Desagher S, Martinou JC. Mitochondria as the central control point of apoptosis. Trends Cell Biol 2000; 10: 369-377.

19. Youle RJ, Karbowski M. Mitochondrial fission in apoptosis. Nat Rev Mol Cell Biol 2005; 6 : 657-663.

20. Haga N, Fujita N, Tsuruo T. Mitochondrial aggregation precedes cytochrome $c$ release from mitochondria during apoptosis. Oncogene 2003; 22: 5579-5585.

21. Leng RP, Lin $Y, M a W$, Wu H, Lemmers B, Chung $S$ et al. Pirh2, a p53-induced ubiquitinprotein ligase, promotes p53 degradation. Cell 2003; 112: 779-791.
22. Duan S, Yao Z, Hou D, Wu Z, Zhu WG, Wu M. Phosphorylation of Pirh2 by calmodulindependent kinase II impairs its ability to ubiquitinate p53. EMBO J 2007; 26: 3062-3074.

23. Herrlich $\mathrm{P}$, Ponta $\mathrm{H}$, Rahmsdorf HJ. DNA damage-induced gene expression: signal transduction and relation to growth factor signaling. Rev Physiol Biochem Pharmacol 1992; 119: 187-223

24. Derijard B, Hibi M, Wu IH, Barrett T, Su B, Deng T et al. JNK1: a protein kinase stimulated by UV light and Ha-Ras that binds and phosphorylates the c-Jun activation domain. Cell 1994; 76: 1025-1037.

25. Heggeness MH, Simon M, Singer SJ. Association of mitochondria with microtubules in cultured cells. Proc Natl Acad Sci USA 1978; 75: 3863-3866.

26. Terasaki M, Chen LB, Fujiwara K. Microtubules and the endoplasmic reticulum are highly interdependent structures. J Cell Biol 1986; 103: 1557-1568.

27. Kim S, Coulombe PA. Intermediate filament scaffolds fulfill mechanical, organizational, and signaling functions in the cytoplasm. Genes Dev 2007; 21: 1581-1597.

28. Ku NO, Darling JM, Krams SM, Esquivel CO, Keeffe EB, Sibley RK et al. Keratin 8 and 18 mutations are risk factors for developing liver disease of multiple etiologies. Proc Natl Acad Sci USA 2003; 100: 6063-6068.

29. Ku NO, Strnad P, Zhong BH, Tao GZ, Omary MB. Keratins let liver live: mutations predispose to liver disease and crosslinking generates Mallory-Denk bodies. Hepatology 2007; 46: 1639-1649.

30. Chedid A, Mendenhall CL, Tosch T, Chen T, Rabin L, Garcia-Pont P et al. Significance of megamitochondria in alcoholic liver disease. Gastroenterology 1986; 90: 1858-1864.

31. Barbaro G, Di Lorenzo G, Asti A, Ribersani M, Belloni G, Grisorio B et al. Hepatocellular mitochondrial alterations in patients with chronic hepatitis $\mathrm{C}$ : ultrastructural and biochemical findings. Am J Gastroenterol 1999; 94: 2198-2205.

32. Caldwell SH, Swerdlow RH, Khan EM, lezzoni JC, Hespenheide EE, Parks JK et al. Mitochondrial abnormalities in non-alcoholic steatohepatitis. J Hepatol 1999; 31: 430-434.

33. Kumemura $\mathrm{H}$, Harada M, Yanagimoto $\mathrm{C}$, Koga H, Kawaguchi $\mathrm{T}$, Hanada $\mathrm{S}$ et al. Mutation in keratin 18 induces mitochondrial fragmentation in liver-derived epithelial cells. Biochem Biophys Res Commun 2008; 367: 33-40. 